\title{
GEICO1601-ROLANDO: a multicentric single arm Phase II clinical trial to evaluate the combination of olaparib and pegylated liposomal doxorubicin for platinum-resistant ovarian cancer
}

J Alejandro Perez-Fidalgo*,1, Maria Iglesias², Uriel Bohn ${ }^{3}$, Elisa Calvo ${ }^{4}$, Yolanda Garcia ${ }^{5}$, Eva Guerra ${ }^{6}$, Luis Manso ${ }^{7}$, Ana Santaballa ${ }^{8}$ \& Antonio Gonzalez-Martin ${ }^{9}$

${ }^{1}$ Department of Medical Oncology, Hospital Clinico Universitario de Valencia, CIBERONC, Spain

2Department of Medical Oncology, Hospital Universitario Son Llatzer, Palma de Mallorca, Spain

${ }^{3}$ Department of Medical Oncology, Hospital Universitario Dr Negrin, Las Palmas de Gran Canaria, Spain

${ }^{4}$ Department of Medical Oncology, Hospital Universitario Virgen del Rocio, Sevilla, Spain

${ }^{5}$ Department of Medical Oncology, Hospital Universitario Parc Tauli, Sabadell, Spain

${ }^{6}$ Department of Medical Oncology, Hospital Universitario 12 de Octubre, Madrid, Spain

${ }^{7}$ Department of Medical Oncology, Hospital Universitario Ramon y Cajal, Madrid, Spain

${ }^{8}$ Department of Medical Oncology, Hospital Politecnico y Universitario La Fe, Valencia, Spain

${ }^{9}$ Department of Medical Oncology, Clinica Universitaria de Navarra, Madrid, Spain

*Author for correspondence: japfidalgo@msn.com

Response to polyadenosine diphosphate ribose polymerase (PARP) inhibitors in platinum-resistant ovarian cancer and in the absence of BRCA mutations is very low. Combining PARP inhibitors with other agents might overcome this lack of activity. Here we describe the rationale and design of GEICO1601-ROLANDO (resistant ovarian cancer treated with olaparib and pegylated liposomal doxorubin; NCT03161132). ROLANDO is a Phase II single-arm multicenter trial in which patients are treated with a combination of olaparib and pegylated liposomal doxorubicin (PLD) in platinum-resistant epithelial ovarian, primary peritoneal, or Fallopian tube cancer regardless of the BRCA mutation status. The primary end point is progression-free survival at 6 months. Other secondary end points are response rate, disease control rate, quality of life and overall survival.

Lay abstract: PARP inhibitors as a single agent have shown very modest activity in platinum-resistant ovarian cancer in a BRCA nonselected population. The GEICO1601-ROLANDO trial is a protocol designed with the aim of assessing efficacy and safety of the combination of olaparib and pegylated liposomal doxorubin followed by olaparib maintenance in this setting.

First draft submitted: 18 October 2018;; Accepted for publication: 12 December 2018; Published online: 10 January 2019

Keywords: maintenance $\bullet$ olaparib • ovarian cancer $\bullet$ pegylated liposomal doxorubicin $\bullet$ platinum-resistant

Ovarian cancer is a leading cause of death, being the fourth most frequent cause of mortality in women in developed countries [1].

Family history and the presence of mutations in genes such as BRCA1 and BRCA2 significantly increase the risk of development of ovarian cancer; other factors such as hormonal or reproductive factors can either increase or decrease the likelihood of this disease [2].

The overall median survival for ovarian cancer is about $40-50 \%$ at 10 years from diagnosis. However, this falls significantly to $21 \%$ and $<6 \%$ when the disease is diagnosed at Stages III and IV, respectively. Approximately $90 \%$ of ovarian cancers are epithelial in origin, of which $60-70 \%$ are high-grade serous ovarian cancer [3].

Epithelial ovarian cancer is highly chemosensitive and the current standard of care for newly diagnosed cases involves debulking surgery and adjuvant platinum- and taxane-based combination chemotherapy. Although most 
Table 1. Trials with olaparib in platinum-resistant ovarian cancer harboring BRCA mutations.

\begin{tabular}{|c|c|c|c|c|c|}
\hline Study (year) [Ref.] & Design & $n$ & Setting & $\begin{array}{l}\text { Response rate/clinical } \\
\text { benefit rate }\end{array}$ & Platinum-resistant relapse \\
\hline
\end{tabular}

b.i.d.: Two-times a day; CBR: Clinical benefit rate; CR: Complete response; ORR: Overall response rate; PR: Partial response.

patients achieve a remission with front-line therapy, unfortunately, up to $80 \%$ of patients will relapse [4]. Disease recurring $>6$ months from the last platinum-based chemotherapy is considered as platinum-sensitive relapse while recurrences within the first 6 months are classified as platinum resistant. Platinum sensitive is more likely to respond to platinum-based chemotherapy; however, the development of platinum resistance is an inevitable event [5].

The development of PARP inhibitors for the treatment of tumors deficient in BRCA1 or BRCA2 is driven by the concept of synthetic lethality, a phenomenon in which the individual deletion of two independent genes does not cause cell death, but the combined deletion is cytotoxic.

Olaparib (AZD2281) is an oral PARP-1 and -2 inhibitor largely tested among ovarian cancer patients. In the Phase I clinical trial patients with different solid tumors refractory to standard treatment were included. Partial response and clinical benefit were observed in those patients harboring BRCA mutation diagnosed with breast, ovarian and prostate cancer [6].

The expansion cohort of this Phase I trial finally included $50 \mathrm{BRCA}$ mutation carriers with platinum-refractory (26\%), platinum-resistant (48\%) and platinum-sensitive relapse (26\%) [7]. Olaparib, at different dose levels, achieved a $28 \%$ of response rates by response evaluation criteria in solid tumors (RECIST; 95\% CI: 16.2-42.5\%), 46.2\% in platinum-sensitive and $33.5 \%$ in platinum-resistant recurrences, with no responses in the platinum-refractory group. Clinical benefit was also meaningful with $46 \%$ in the whole series, which was particularly relevant in the platinum-sensitive group $(69.2 \%)$, but also interesting in the platinum-resistant $(45.8 \%)$ and -refractory $(23.1 \%)$ groups.

A later Phase II trial finally confirmed the activity of olaparib in monotherapy in a heavily pretreated $g B R C A m u t$ ovarian cancer population. In this international study, patients irrespective of interval of platinum were treated after a median of three previous lines in two different cohorts with olaparib $400 \mathrm{mg}$ two-times a day (b.i.d.) and $100 \mathrm{mg}$ b.i.d. Overall response rate (ORR) was 30\% in the cohort treated with $400 \mathrm{mg}$ and $13 \%$ in the cohort with olaparib $100 \mathrm{mg}$. The most frequent adverse events were nausea, fatigue and anemia, which were slightly superior in the cohort of $400 \mathrm{mg}$ b.i.d. [8].

In 2012, a multicentre Phase II study by Kaufman et al. enrolled individuals with a germline BRCA1/2 mutation and recurrent cancer. Eligibility included ovarian cancer resistant to prior platinum; breast cancer with more than or equal to three chemotherapy regimens for metastatic disease; pancreatic cancer with prior gemcitabine treatment; or prostate cancer with progression on hormonal and one systemic therapy. Olaparib was administered as a single agent at $400 \mathrm{mg}$ b.i.d. The primary efficacy end point was tumor response rate. A total of 298 patients received treatment. The tumor response rate was $31.1 \%$ in the ovarian cancer cohort (all platinum resistant; Tables $1 \&$ 2) [9].

Based on the study by Kaufman et al., olaparib was approved by the US FDA for refractory, advanced ovarian cancer associated with germline breast cancer susceptibility gene (BRCA) mutations in 2014. In August 2017, olaparib was additionally approved by the FDA as a maintenance therapy for women with recurrent epithelial ovarian, fallopian tube or primary peritoneal cancer who have achieved complete or partial response to platinumbased chemotherapy, irrespective of their BRCA status.

However, EMA approval of olaparib has been limited to platinum-sensitive relapse. 


\begin{tabular}{|c|c|c|c|c|c|c|}
\hline Study (year) [Ref.] & Design & $\mathbf{n}$ & Setting & $\begin{array}{l}\text { Response rate in } \\
\text { overall population }\end{array}$ & $\begin{array}{l}\text { Response rate in } \\
B R C A \text {-mutated } \\
\text { population }\end{array}$ & $\begin{array}{l}\text { Response rate in } B R C A \\
\text { wt population }\end{array}$ \\
\hline $\begin{array}{l}\text { Gelmon et al. } \\
\text { (2011) [10] }\end{array}$ & $\begin{array}{l}\text { Phase II single arm } \\
\text { (olaparib } 400 \text { b.i.d.). } \\
\text { End point: response }\end{array}$ & $\begin{array}{l}91 \\
\text { ( } 65 \text { ovarian } \\
\text { cancer) } \rightarrow 17 \text { BRCA } \\
\text { mut and } 47 \text { BRCA no } \\
\text { mut }\end{array}$ & $\begin{array}{l}\text { Recurrent ovarian } \\
\text { cancer. } \\
\text { Median } 3 \text { previous } \\
\text { lines }(1-10)\end{array}$ & OR $29 \%$ & $\begin{array}{l}60 \% \\
(3 / 5 \text { platinum } \\
\text { sensitive); } \\
33 \% \\
(4 / 12 \text { platinum } \\
\text { resistant) }\end{array}$ & $\begin{array}{l}50 \% \\
(10 / 20 \text { platinum } \\
\text { sensitive); } \\
4 \% \\
\text { ( } 1 / 26 \text { platinum } \\
\text { resistant) }\end{array}$ \\
\hline
\end{tabular}

Olaparib was compared with pegylated liposomal doxorubicin (PLD) in a multicenter randomized Phase II trial. Patients with BRCA1 or BRCA2 germline mutations recurring within 12 months of prior platinum therapy were randomized to receive olaparib $200 \mathrm{mg}$ b.i.d. versus olaparib $400 \mathrm{mg}$ b.i.d. versus PLD $50 \mathrm{mg} / \mathrm{m}^{2}$ intravenously every 28 days [11]. 97 patients were included, of whom $42.4-56.4 \%$ were platinum resistant. Of note, the proportion of platinum-resistant relapses was inferior in the PLD arm (42.4\%). Progression-free survival (PFS) was 6.5 months (95\% CI: 5.5-10.1 months), 8.8 months (95\% CI: 5.4-9.2 months) and 7.1 months (95\% CI: 3.7-10.7 months) for olaparib $200 \mathrm{mg}$, olaparib $400 \mathrm{mg}$ and PLD, respectively, with an unexpectedly high PFS for the PLD arm. There were no statistically significant differences between combined arms of single agent olaparib versus PLD (HR: 0.88; 95\% CI: 0.51-1.56; $\mathrm{p}=0.66$ ). RECIST-assessed response rates were 25,31 and $18 \%$, respectively, with no statistically significant differences. This study showed a similar activity and a trend toward improvement with olaparib versus PLD in patients with relapsed ovarian cancer and BRCA1 or BRCA2 mutations.

Although olaparib has been tested in combination to chemotherapy with carboplatin and paclitaxel in a randomized Phase II trial [12], the only previous experience of a combination of PLD and concurrent olaparib comes from a Phase I trial [13].

\section{The ROLANDO trial}

Here we describe the rationale and design of ROLANDO, a single-arm Phase II trial, designed to evaluate the efficacy and safety of the combination of olaparib plus PLD in platinum-resistant epithelial ovarian cancer.

\section{Background \& rationale}

The combination of olaparib and liposomal doxorubicin in ovarian cancer has been tested previously in a Phase I trial [13]. 44 patients with ovarian $(n=28)$, breast $(n=13)$ and other solid tumors $(n=3)$ were treated with olaparib continuously (days $1-28$ ) or intermittently (days $1-7)$ plus PLD $\left(40 \mathrm{mg} / \mathrm{m}^{2}\right.$ day 1$)$. Seven olaparib cohorts were explored (50-400 mg b.i.d.) to determine the recommended dose. Olaparib $400 \mathrm{mg}$ b.i.d. plus PLD was well tolerated and no dose lethal toxicity was detected. Grade 3 adverse events (AE) were reported in 27 patients (61\%), and serious $\mathrm{AE}$ occurred in 12 patients (27\%). Most frequent Grade $3 \mathrm{AE}$ were stomatitis, nausea and asthenia, which happened mainly in the cohort of olaparib $400 \mathrm{mg}$ b.i.d. continuously.

No major interactions were observed between PLD and olaparib. The ORR was 33\% (14 out of 42 patients, three complete responses). A total of 13 responders had ovarian cancer (response rate $46.4 \%$ in ovarian cancer), of whom ten were platinum sensitive.

Recently, a preclinical study demonstrated a synergism of the combination of olaparib with doxorubicin in 2D and spheroid models of ovarian cancer [14]. This study showed that DNA damage was increased at a molar concentration of doxorubicin: olaparib of 50:1 in both $B R C A$ mutant and $B R C A$ wild type cell lines UWB1-289. The combination of PLD and olaparib resulted in a significantly increased number of double-strand breaks assessed by $\gamma H 2 A X$ assay compared with olaparib single agent (approximately 10- and 13-fold increases for UWB1.289 and UWB1.289 +BRCA, respectively) and to doxorubicin single agent (approximately 50 and $65 \%$ increases, respectively).

\section{Design}

Study design

GEICO 1601-ROLANDO (NCT03161132) is a single-arm prospective trial to assess the efficacy and safety of the combination of olaparib and PLD in the platinum-resistant setting. 


\section{Table 3. Key inclusion and exclusion criteria.}

\begin{tabular}{ll} 
Key inclusion criteria & Key exclusion criteria \\
$\begin{array}{l}\text { Platinum-resistant recurrent high-grade serous or endometrioid ovarian cancer, } \\
\text { primary peritoneal or fallopian tube cancer ( }>28 \text { days and }<6 \text { months since } \\
\text { previous platinum) }\end{array}$ & $\begin{array}{l}\text { Platinum-refractory ovarian cancer (relapse during or }<28 \text { days since last } \\
\text { platinum-containing chemotherapy) }\end{array}$ \\
\hline$\leq 3$ prior chemotherapy regimens & Previous therapy with PARP inhibitor \\
\hline $\begin{array}{l}\text { Any } B R C A \text { status: } \\
-B R C A \text { wt or unknown: at least one previous platinum-sensitive relapse }\end{array}$ & $\begin{array}{l}\text { Previous therapy with PLD except if }>6 \text { months since last PLD and given as a part of } \\
\text { a platinum-sensitive relapse }\end{array}$ \\
\hline ECOG $\leq 2$ & LVEF less than threshold of normality \\
\hline Measurable disease according to RECIST 1.1 & \\
\hline Adequate renal, liver and bone marrow function (Hb $\geq 10 \mathrm{~g} / \mathrm{dl})$ & \\
\hline $\begin{array}{l}\text { ECOG: Eastern Cooperative Oncology Group; Hb: Hemoglobin; LVEF: Left ventricular ejection fraction; PLD: Pegylated liposomal doxorubicin; RECIST: Response evaluation criteria in solid } \\
\text { tumors. }\end{array}$ &
\end{tabular}

Pegylated liposomal doxorubicin $40 \mathrm{mg} / \mathrm{m}$ every 28 days $\times$ six cycles

Olaparib 300 mg b.i.d. (tablets)

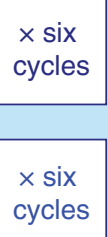
maintenance

Figure 1. Treatment schedule of the GEICO1601-ROLANDO trial.

b.i.d.: Twice daily.

\section{Primary \& secondary objectives}

Primary efficacy end point is 6 months PFS rate (PFS6m) of the combination of olaparib $300 \mathrm{mg}$ b.i.d. in tablet formulation + PLD $40 \mathrm{mg} / \mathrm{m}^{2}$ intravenously every 28 days followed by olaparib $300 \mathrm{mg}$ b.i.d. tablet formulation in platinum-resistant relapsed ovarian cancer patients.

Secondary end points are safety and objective response rate (ORR), defined as the sum of complete and partial responses by RECIST 1.1, disease-control rate (complete response, partial response or stable disease), CA-125 response, PFS, postprogression survival, overall survival, quality of life and growth-modulation index.

\section{Key eligibility criteria}

Key eligibility criteria are shown in Table 3.

\section{Planned sample size}

The sample size was determined based on exact single-stage Phase II design. We assumed that a new combination with 6-month PFS of $15 \%$ or less would be considered of little clinical significance and that an agent with PFS $40 \%$ or higher would be of interest for further investigation. With $90 \%$ power and at a two-sided 0.05 significance level, 27 evaluable patients should be included in the study, requiring at least ten patients surviving after 6 months to reject the null hypothesis.

In order to obtain the required evaluable number of patients we expect to recruit 32 patients ( $20 \%$ dropouts).

\section{Dose \& schedule of therapy}

This is a single arm trial in which patients will be treated with a combination of PLD and olaparib for six cycles following maintenance therapy with olaparib (Figure 1). 


\section{Efficacy evaluation}

For efficacy assesment subsequent CT scan tumor evaluation according to RECIST 1.1 criteria will be performed systematically every 8 weeks. Tumor assessment will also be done at planned intervals. Visits will include systematically only physical examination and CA-125 analysis.

Patients must have been treated with at least two cycles in order to be considered evaluable. After six cycles of PLD plus olaparib, the patient will be assessed for response to treatment, and only those patients achieving response or stable disease will go on to the maintenance phase.

Disease follow-up will be done according to RECIST criteria V1.1, computed tomography scans, which should be performed at screening and every 8 weeks during the first 12 months and every 12 weeks after 12 months from start of treatment, both until disease progression.

Also, CA-125 levels will be followed, CA-125 determinations should be performed at screening and every 8 weeks during the first 12 months and every 12 weeks after 12 months from start of treatment, both until disease progression. Rising of CA-125 will not be considered progression until availability of confirmatory computed tomography scan.

\section{Safety evaluation}

Patients will be visited according the following schema:

- Every week during the first month of treatment.

- Every 2 weeks during the second month of treatment.

- Every 4 weeks from 3 up to 12 months (calculated from first study treatment administration).

- Every 12 weeks up to 2 years calculated from first study treatment administration.

- According to local normal clinical practice from 2 years until exit.

- At the end of study treatment.

- Safety visit (after 30 days of end of treatment).

AE will be graded according to National Cancer Institute - Common Terminology Criteria for Adverse Events (NCI CTCAE) V4.03. AE G $\geq 2$ will be recorded at each visit during treatment until the safety visit.

Safety visit will be performed at day $30+/-3$ days after end of treatment in order to determine patient general status.

\section{Statistical analysis}

Demographics and baseline characteristics will be summarized using descriptive statistics. For categorical variables, the number (n) and percent of each category within a parameter will be presented. For continuous variables, the sample size (n), mean, median and standard deviation, as well as the minimum and maximum values, will be determined.

All efficacy analyses will be based primarily on the intention-to-treat analysis set and secondarily on the per protocol analysis set, which are defined as follows:

- Intention-to-treat analysis set: all patients participating in the study receiving at least a single dose of study medication.

- Per protocol analysis set: all patients participating in the study who fulfill the protocol in terms of the eligibility, interventions and outcome assessment receiving at least two cycles of DLP + olaparib.

The primary end point of PFS6m will be estimated using Kaplan-Meier method.

The secondary end points of ORR defined as the proportion of patients with a confirmed complete response or partial response as per RECIST 1.1, and the disease control rate defined as the sum of complete response + partial response + stable disease will be estimated along with a 95\% CI.

Additional secondary end points of PFS, postprogression survival and overall survival (as assessed by the investigator) will be estimated using Kaplan-Meier method.

\section{Recruiting sites}

The study is enrolling patients in eight institutions in Spain. 


\section{Conclusion}

The GEICO 1601-ROLANDO trial is a study that aims to respond to the question of whether the combination of PLD and olaparib has a role in the platinum-resistant setting irrespective of $B R C A$ mutation status.

\section{Executive summary}

- PARP inhibitors (PARPi) have shown important activity in platinum-sensitive relapse and BRCA-mutated ovarian cancer. However, activity of PARPi is modest in platinum-resistant relapse and BRCA-unknown or not mutated patients.

- The GEICO1601-ROLANDO trial is a single-arm Phase II trial designed to evaluate the efficacy and safety of the combination of olaparib and pegylated liposomal doxorubicin (PLD) in platinum-resistant ovarian cancer irrespectively of BRCA mutation status.

- Primary end point is progression-free survival (PFS) at 6 months.

- Secondary end points are safety, overall response rate, disease-control rate, CA-125 response, PFS and overall survival.

- Among the key inclusion criteria are high-grade serous or endometrioid ovarian carcinoma, platinum resistance relapse defined as $>28$ days and $<6$ months since last platinum-containing schedule, no more than three previous lines, if $B R C A$ status is unknown or nonmutated at least one previous platinum-sensitive relapse is required, if $B R C A$ is mutated primary platinum resistance is allowed, an Eastern Cooperative Oncology Group less than or equal to two, measurable disease and appropriate bone marrow and renal and hepatic function. Previous PLD is allowed if given during a platinum-sensitive relapse and $>6$ months. Previous PARPi is excluded.

- PLD will be administered at $40 \mathrm{mg} / \mathrm{m}^{2}$ every 28 days in combination with olaparib tablets $300 \mathrm{mg} / 12 \mathrm{~h}$ continuously for six cycles followed by maintenance with olaparib. The planned sample size was calculated assuming that PFS6m $15 \%$ or less will be clinically futile and that PFS6m $40 \%$ or higher is clinically relevant. Sample size needed with $20 \%$ dropouts is 32 patients.

- To date 16 patients have been recruited; results are expected for the end of 2019.

Supplementary data

An infographic accompanies this paper at the end of the references section. To download the infographic that accompanies this paper, please visit the journal website at: www.future-science.com/doi/full/10.4155/fsoa-2018-0107

Author contributions

JA Perez-Fidalgo and A Gonzalez-Martin designed the study, wrote manuscript and approved final manuscript. M Iglesias, U Bohn, W Calvo, Y Garcia, E Guerra, L Manso and A Santaballa wrote manuscript and approved final manuscript.

Financial \& competing interests disclosure

This trial received financial support from AstraZeneca. JA Perez-Fidalgo discloses lectures, advisories or traveling support from AstraZeneca, Roche, Pharmamar, Clovis and Pfizer. E Guerra discloses a role as advisory board for AstraZeneca. L Manso discloses lectures, advisories/consultant role and research funding from Tesaro, AstraZeneca, Roche, Novartis and Celgene. A GonzalezMartin discloses lectures, advisories or traveling support from AstraZeneca, Roche, Tesaro, Clovis, Pfizer-Merck and Pharmamar. The rest of the authors have no disclosures to declare. The authors have no other relevant affiliations or financial involvement with any organization or entity with a financial interest in or financial conflict with the subject matter or materials discussed in the manuscript apart from those disclosed.

No writing assistance was utilized in the production of this manuscript.

\section{Ethical conduct of research}

The authors state that they have obtained appropriate institutional review board approval or have followed the principles outlined in the Declaration of Helsinki for all human or animal experimental investigations. In addition, for investigations involving human subjects, informed consent has been obtained from the participants involved.

Open access

This work is licensed under the Creative Commons Attribution 4.0 License. To view a copy of this license, visit: http://creativeco mmons.org/licenses/by/4.0/ 


\section{References}

1. Jayson GC, Kohn E, Kitchener HC, Ledermann JA. Ovarian cancer. Lancet 384(9951), 1376-1388 (2014).

2. Reid BM, Permuth JB, Seller TA. Epidemiology of ovarian cancer: a review. Cancer Biol. Med. 14(1), 9-32 (2017).

3. Colombo PE, Fabbro M, Theillet C, Bibeau F, Rouanet P, Ray-Coquard I. Sensitivity and resistance to treatment in the primary management of epithelial ovarian cancer. Crit. Rev. Oncol. Hematol. 89(2), 207-216 (2014).

4. Desai A, Xu J, Aysola K et al. Epithelial ovarian cancer: an overview. World J. Transl. Med. 3(1), 1-8 (2014).

5. Markman M, Bookman MA. Second-line treatment of ovarian cancer. Oncologist 5(1), 25-35 (2000).

6. Fong PC, Boss DS, Yap TA et al. Inhibition of poly (ADP-ribose) polymerase in tumors from BRCA-carriers. N. Eng. J. Med. 361, 123-134 (2009).

7. Fong PC, Yap TA, Boss DS et al. Poly (ADP-ribose) polymerase inhibition: frequent durable responses in BRCA carrier ovarian cancer correlating with platinum-free interval. J. Clin. Oncol. 28, 2512-2519 (2010).

8. Audeh MH, Carmichael J, Penson RT et al. Oral poly-ADP ribose polymerase inhibitor olaparib in patients with BRCA1 or BRCA2 mutations in recurrent ovarian cancer: a proof-of-concept trial. Lancet Oncol. 376, 245-251 (2010).

9. Kaufman B, Shapira-Frommer R, Schmutzler RK et al. Olaparib monotherapy in patients with advanced cancer and a germline BRCA1/2 mutation. J. Clin. Oncol. 33(3), 244-250 (2015).

10. Gelmon KA, Tischkowitz M, Mackay $\mathrm{H}$ et al. Olaparib in patients with recurrent high-grade serous or poorly differentiated ovarian carcinoma or triple-negative breast cancer: a Phase II, multicentre, open-label, non-randomised study. Lancet Oncol. 12(9), 852-861 (2011).

11. Kaye SB, Lubinsky J, Matulonis U et al. Phase II open-label, randomized multicenter study comparing the efficacy and safety of olaparib, a poly (ADP-ribose) polymerase inhibitor and pegylated liposomal doxorubicin in patients with $B R C A 1$ or $B R C A 2$ mutations and recurrent ovarian cancer. J. Clin. Oncol. 30, 372-379 (2011).

12. Oza AM, Cibula D, Oaknin A et al. Olaparib combined with chemotherapy for recurrent platinum-sensitive ovarian cancer: a randomized Phase II trial. Lancet Oncol. 16, 87-97 (2015).

13. Del Conte G, Sessa C, Von Moos R et al. Phase I study of olaparib in combination with liposomoal doxorubicin in patients with advanced solid tumors. B. J. Cancer 111, 651-659 (2014).

14. Eetezadi S, Evans JC, Shen Y-T, De Souza R, Piquette-Miller M, Allen C. Ratio-dependent synergism of doxorubicin and olaparib combination in 2D and spheroid models of ovarian cancer. Mol. Pharm. 15, 472-485 (2018). 
